\title{
Core-Level Photoelectron Spectroscopy Probing Local Strain at Silicon Surfaces and Interfaces
}

\author{
Oleg V. Yazyev* \\ Ecole Polytechnique Fédérale de Lausanne (EPFL), \\ Institute of Chemical Sciences and Engineering, CH-1015 Lausanne, Switzerland \\ Alfredo Pasquarello \\ Ecole Polytechnique Fédérale de Lausanne (EPFL), \\ Institute of Theoretical Physics, CH-1015 Lausanne, Switzerland and \\ Institut Romand de Recherche Numérique en Physique des Matériaux (IRRMA), CH-1015 Lausanne, Switzerland
}

(Dated: October 26, 2018)

\begin{abstract}
Using a first-principles approach, we investigate the origin of the fine structure in Si $2 p$ photoelectron spectra at the $\mathrm{Si}(100) 2 \times 1$ surface and at the $\mathrm{Si}(100)-\mathrm{SiO}_{2}$ interface. Calculated and measured shifts show very good agreement for both systems. By using maximally localized Wannier functions, we provide an interpretation in which the effects due to the electronegativity of second nearest neighbor atoms and due to the local strain field are distinguished. Hence, in combination with accurate modeling, photoelectron spectroscopy can provide a direct measure of the strain field at the atomic scale.
\end{abstract}

PACS numbers: 79.60.-i,73.20.-r,68.35.-p

The increasing availability of synchrotron radiation facilities is bearing X-ray photoemission spectra of unprecedented resolution characterizing surfaces and interfaces [1]. The achieved sensitivity is sufficient to distinguish inequivalent subsurface atoms with identically composed first-neighbor shells. Hence, the interpretation of such core-level spectra can no longer be achieved with simple electronegativity arguments, but requires the consideration of the interplay between local strain fields and electronegativity effects of second-nearest neighbors.

These difficulties are strikingly illustrated for the $\operatorname{Si}(100) 2 \times 1$ surface and for the technologically relevant $\mathrm{Si}(100)-\mathrm{SiO}_{2}$ interface, which have been the objects of numerous highly resolved X-ray photoemission investigations. While the shifts pertaining to the first-layer dimer atoms of the $\mathrm{Si}(100) 2 \times 1$ surface have been identified, the other lines appearing in highly resolved Si $2 p$ spectra still lack a consensual assignment [2, 3, 4]. For the $\mathrm{Si}(100)-\mathrm{SiO}_{2}$ interface, highly resolved spectra show fine structure in the nonoxidized $\mathrm{Si}$ line, with extra components at lower $\left(\mathrm{Si}^{\alpha}\right)$ and higher binding energy $\left(\mathrm{Si}^{\beta}\right)$ with respect to the Si bulk line [5, 6] .

We investigate the origin of the fine structure in $\mathrm{Si}$ $2 p$ photoemission spectra at silicon surfaces and interfaces using density functional theory calculations based on pseudopotentials (PPs) and plane waves [7]. To interpret the Si $2 p$ photoelectron spectra at silicon surfaces and interfaces, it is necessary to evaluate core-level shifts with respect to the Si bulk line. Since these shifts mainly result from the relaxation of valence electron states, their accurate determination is possible within a PP scheme, which does not treat core electrons explicitly 8,9 ]. We calculated Si $2 p$ shifts including the effect of core-hole relaxation by taking total energy differences between two
TABLE I: Comparison between calculated and measured $\mathrm{Si}$ $2 p$ shifts at the $\mathrm{Si}(001)-c(4 \times 2)$ surface. The shifts (in $\mathrm{eV})$ are given with respect the $\mathrm{Si}$ bulk line. The meaning of labels is explained in Fig. 1

\begin{tabular}{ccccc}
\hline \hline & $\begin{array}{c}\text { Theory } \\
\text { Present }\end{array}$ & Ref. [2] & $\begin{array}{c}\text { Experiment } \\
\text { Ref. [3] }\end{array}$ & Ref. [4] \\
\hline $1 u$ & -0.49 & -0.49 & -0.49 & -0.50 \\
$1 d$ & 0.02 & 0.06 & 0.06 & 0.06 \\
2 & -0.01 & & & \\
3 & -0.14 & -0.21 & -0.20 & -0.21 \\
4 & -0.26 & & & \\
$3^{\prime}$ & 0.24 & 0.22 & 0.20 & 0.23 \\
$4^{\prime} u$ & 0.21 & & & \\
$4^{\prime} d$ & 0.10 & & & \\
\hline \hline
\end{tabular}

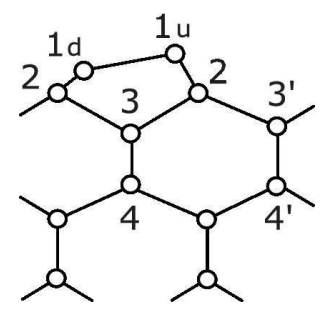

FIG. 1: The adopted labeling of $\mathrm{Si}$ atoms at the $\mathrm{Si}(001)$ $c(4 \times 2)$ surface. The $4^{\prime}$ atoms can be further separated into inequivalent up $\left(4^{\prime} u\right)$ and down $\left(4^{\prime} d\right)$ atoms.

separate self-consistent calculations. First, the groundstate energy is determined; then the $\mathrm{PP}$ of a given $\mathrm{Si}$ atom is replaced by another $\mathrm{PP}$ which simulates the presence of a screened $2 p$ hole in its core [8].

For the $\mathrm{Si}(100)-\mathrm{c}(4 \times 2)$ surface we find binding energies of $-0.49 \mathrm{eV}$ and $0.02 \mathrm{eV}$ for dimer-up and dimer-down atoms, in good agreement with experimental results [2, $\underline{3}$, 


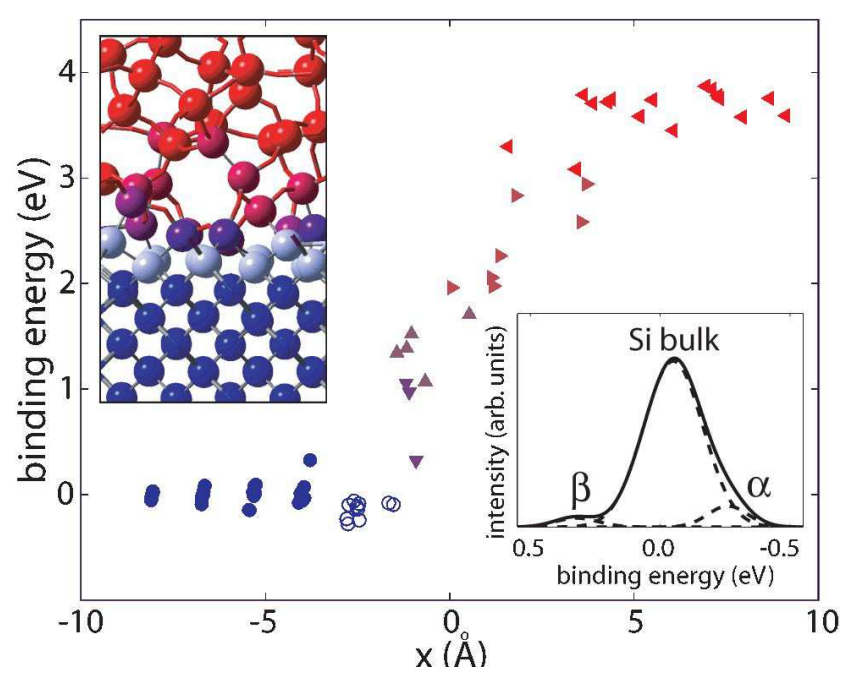

FIG. 2: Calculated $\mathrm{Si} 2 p$ shifts at the $\mathrm{Si}(100)-\mathrm{SiO}_{2}$ interface along an orthogonal direction to the interface. The $\mathrm{Si}^{+n}$ correspond to oxidized $\mathrm{Si}$ atoms with $n \mathrm{O}$ neighbors $(n=1-\boldsymbol{\nabla}$, $2-\boldsymbol{\Delta}, 3-\boldsymbol{\Delta}, 4-\boldsymbol{\Delta})$ and are color-coded in the atomistic model. Nonoxidized $\mathrm{Si}$ atoms $\left(\mathrm{Si}^{0}\right)$ with (o) and without $(\bullet)$ second-neighbor $\mathrm{O}$ atoms are distinguished. Inset: simulated spectrum for $\mathrm{Si}^{0}$ (Gaussian broadened: $\sigma=0.08 \mathrm{eV}$ ) and its decomposition in three components.

TABLE II: Comparison between calculated and measured Si $2 p$ shifts at the $\mathrm{Si}(100)-\mathrm{SiO}_{2}$ interface. The $\mathrm{Si}$ bulk line is taken as reference.

\begin{tabular}{cccc}
\hline \hline & Theory & \multicolumn{2}{c}{ Experiment } \\
& Present & Ref. [5] & Ref. [6] \\
\hline$\alpha$ & -0.21 & -0.25 & -0.22 \\
$\beta$ & 0.32 & 0.20 & 0.34 \\
+1 & 0.78 & 1.00 & 0.95 \\
+2 & 1.40 & 1.82 & 1.78 \\
+3 & 2.37 & 2.62 & 2.60 \\
+4 & 3.64 & 3.67 & 3.72 \\
\hline \hline
\end{tabular}

4]. The second-layer atoms are found to yield very small shifts $(-0.01 \mathrm{eV})$ while third- and fourth-layer atoms give shifts to both lower $(3,4)$ and higher binding energies $\left(3^{\prime}\right.$, $\left.4^{\prime}\right)$ with respect to the Si bulk line (Table 【). Shifts of deeper layers are negligible.

For the $\mathrm{Si}(100)-\mathrm{SiO}_{2}$ interface, we adopt a model structure which incorporates a realistic transition region [10]. Calculated binding energies are given in Fig. 2 along the direction orthogonal to the interface plane. The binding energies of oxidized Si atoms increase almost linearly with oxidation state [9], and show quantitative agreement with experimental values (Table II). The focus of the present investigation is on nonoxidized Si atoms, which give shifts with a significant spread near the interface. The simulated spectrum associated to these $\mathrm{Si}$ atoms could be decomposed into three components (Fig. 2, inset), yielding shifts for the $\mathrm{Si}^{\alpha}$ and $\mathrm{Si}^{\beta}$ lines of $-0.21 \mathrm{eV}$ and $0.32 \mathrm{eV}$, which also agree with experimental data (Table III).

Binding energy shifts of Si atoms with identically composed first-neighbor shells are caused by electron density displacements due to either the local strain fields or electronegativity effects. We carried out an analysis in terms of maximally localized Wannier functions (MLWFs) 11] in order to distinguish between these two effects [12]. For the $\operatorname{Si}(100)-c(4 \times 2)$ surface, we find that, apart from the shifts associated with the dimer atoms, the other shifts mainly result from the local strain induced by the surface reconstruction. For the interface, the $\mathrm{Si}$ atoms with second-neighbor $\mathrm{O}$ atoms contribute to the $\mathrm{Si}^{\alpha}$ line, while the $\mathrm{Si}^{\beta}$ line originates from bond elongations of $\mathrm{Si}$ atoms without second-neighbor $\mathrm{O}$ atoms. From the experimental shift of about $0.3 \mathrm{eV}$, we infer the occurrence of $\mathrm{Si}$ atoms with an average bond length elongation of $\sim 0.05 \AA$.

In conclusion, we revealed the physical mechanisms underlying the fine structure in Si $2 p$ photoelectron spectra at silicon surfaces and interfaces. Maximally localized Wannier functions offer a powerful tool to identify corelevel shifts originating from the electronegativity of farther neighbors. A key result of our work is then that the remaining lines provide an atomic-scale probe of the strain in the structure. This confers to photoelectron spectroscopy a new functionality in addition to the detection of chemical composition.

* Electronic address: oleg.yazyev@epfl.ch

[1] F. J. Himpsel et al., in Proceedings of the International School of Physics Enrico Fermi, edited by M. Campagna and R. Rosei (Elsevier, Amsterdam, 1990), p. 203-236.

[2] E. Landemark et al., Phys. Rev. Lett. 69, 1588 (1992).

[3] P. De Padova et al., Phys. Rev. Lett. 81, 2320 (1998).

[4] H. Koh et al., Phys. Rev. B 67, 073306 (2003).

[5] J. H. Oh et al., Phys. Rev. B 63, 205310 (2001).

[6] S. Dreiner, M. Schürmann, and C. Westphal, J. Electron Spectrosc. Relat. Phenom. 144-147, 405 (2005).

[7] R. Car and M. Parrinello, Phys. Rev. Lett. 55, 2471 (1985); CPMD version 3.9.1, Copyright IBM Corp 19902004, Copyright MPI für Festkörperforschung Stuttgart 1997-2001, http://www. cpmd.org.

[8] E. Pehlke and M. Scheffler, Phys. Rev. Lett. 71, 2338 (1993).

[9] A. Pasquarello, M. S. Hybertsen, and R. Car, Phys. Rev. Lett. 64, 1024 (1995); Phys. Rev. B 53, 10942 (1996).

[10] A. Bongiorno, A. Pasquarello, M. S. Hybertsen, and L. C. Feldman, Phys. Rev. Lett. 90, 186101 (2003).

[11] N. Marzari and D. Vanderbilt, Phys. Rev. B 56, 12847 (1997).

[12] O. V. Yazyev and A. Pasquarello, Phys. Rev. Lett. 96, 157601 (2006). 